\title{
Angiotensin II infusion and markers of organ function in invasively ventilated COVID-19 patients
}

Alberto Zangrillo, Sergio Colombo, Anna Mara Scandroglio, Evgeny Fominskiy, Marina Pieri, Maria Grazia Calabrò, Paolo Federico Beccaria, Nicola Pasculli, Francesca Guzzo, Maria Rosa Calvi, Antonella Cipriani, Chiara Sartini, Pasquale Nardelli, Alessandro Ortalda, Gaetano Lombardi, Marianna Sartorelli, Giacomo Monti, Andrea Assanelli, Moreno Tresoldi, Lorenzo Dagna, Stefano Franchini, Ary Serpa Neto, Rinaldo Bellomo and Giovanni Landoni

Coronavirus disease 2019 (COVID-19) can cause severe acute respiratory syndrome requiring invasive mechanical ventilation. ${ }^{1}$ Once ventilated, most patients develop vasodilatory hypotension. ${ }^{2}$ The renin-angiotensin-aldosterone system (RAAS) may play an important role in these patients because angiotensin-converting enzyme (ACE) type 2 (ACE2) is the viral receptor 3,4 for the spike protein on the viral surface of severe acute respiratory syndrome coronavirus 2 (SARS-CoV-2) and because its expression may be affected by the use of drugs that inhibit the RAAS. ${ }^{1,5}$

Angiotensin II is a vasopressor for the treatment of catecholamine-resistant vasodilatory shock ${ }^{6}$ and a substrate for ACE2 which has been approved by the United States Food and Drug Administration (FDA) and the European Medicines Agency (EMA). In a phase 2 double blind randomised controlled trial, angiotensin II as rescue vasopressor improved blood pressure in vasodilatory shock, ${ }^{6}$ and increased survival in patients with a high angiotensin I to angiotensin II ratio $^{7}$ and in those with a high renin level. ${ }^{8}$ Moreover, in patients receiving renal replacement therapy (RRT) at randomisation, angiotensin II increased the likelihood of recovery to RRT independence. ${ }^{9}$ Its physiological effect on oxygenation in patients with COVID-19 was recently assessed in an uncontrolled case series which reported an improvement of the arterial partial pressure of oxygen $\left(\mathrm{PaO}_{2}\right)$ to fraction of inspired oxygen $\left(\mathrm{FiO}_{2}\right)$ ratio with angioten $\mathrm{in} 1 \mathrm{II}{ }^{10} \mathrm{In}$ contrast, more recently, in an even smaller case series, investigators from Germany reported that angiotensin II was associated with poor outcomes in patients with COVID-19. ${ }^{11}$ However, this observational assessment also lacked controls.

Given the above findings, we conducted a controlled assessment of the impact of angiotensin II infusion in patients with COVID-19 receiving invasive

\section{ABSTRACT}

Objective: The use of angiotensin $\|$ in invasively ventilated patients with coronavirus disease 2019 (COVID-19) is controversial. Its effect on organ function is unknown.

Design: Prospective observational study.

Setting: Intensive care unit (ICU) of a tertiary academic hospital in Milan, Italy.

Participants: Adult patients receiving mechanical ventilation due to COVID-19.

Interventions: Use angiotensin II either as rescue vasopressor agent or as low dose vasopressor support.

Main outcome measures: Patients treated before angiotensin II was available or treated in an adjacent COVID-19 ICU served as controls. For data analysis, we applied Bayesian modelling as appropriate. We assessed the effects of angiotensin II on organ function.

Results: We compared 46 patients receiving angiotensin II therapy with 53 controls. Compared with controls, angiotensin II increased the mean arterial pressure (median difference, $9.05 \mathrm{mmHg}$; $95 \% \mathrm{Cl}, 1.87-16.22 ; P=0.013$ ) and the $\mathrm{PaO}_{2} / \mathrm{FiO}_{2}$ ratio (median difference, $23.17 ; 95 \% \mathrm{Cl}, 3.46-42.88 ; P=0.021)$, and decreased the odds ratio (OR) of liver dysfunction (OR, 0.32; $95 \% \mathrm{Cl}, 0.09$ 0.94). However, angiotensin II had no effect on lactate, urinary output, serum creatinine, C-reactive protein, platelet count, or thromboembolic complications. In patients with abnormal baseline serum creatinine, Bayesian modelling showed that angiotensin II carried a $95.7 \%$ probability of reducing the use of renal replacement therapy (RRT).

Conclusions: In ventilated patients with COVID-19, angiotensin II therapy increased blood pressure and $\mathrm{PaO}_{2} / \mathrm{FiO}_{2}$ ratios, decreased the OR of liver dysfunction, and appeared to decrease the risk of RRT use in patients with abnormal baseline serum creatinine. However, all of these findings are hypothesis-generating only.

Trial registration: ClinicalTrials.gov NCT04318366.

Crit Care Resusc 2021; 23 (2): 215-224 


\section{ORIGINAL ARTICLES}

mechanical ventilation in a referral centre in Milan, Italy. ${ }^{12}$ After comparison with controls and adjustment for key baseline risk factor imbalances, we aimed to explore whether angiotensin II would affect markers of organ function.

\section{Methods}

\section{Study design}

The COVID-BioB study is an investigation performed at the Istituto di Ricovero e Cura a Carattere Scientifico (IRCCS) San Raffaele Scientific Institute, a 1350-bed university hospital in Milan, Italy. The study was approved by the hospital's Ethics Committee (protocol No. 34/int/2020) and was registered on ClinicalTrials.gov (NCT04318366). The full description of patient management and clinical protocols at San Raffaele was previously published. ${ }^{13}$

\section{Enrolment criteria}

After angiotensin II was obtained from the manufacturer for compassionate use, all patients aged $\geq 18$ years admitted to an intensive care unit (ICU) with confirmed SARS-CoV-2 infection were consecutively enrolled. Confirmed infection was defined as positive real-time reverse-transcriptase polymerase chain reaction (RT-PCR) from a nasal and/or throat swab together with signs, symptoms and radiological findings suggestive of COVID-19 pneumonia. Only patients completing their 28-day follow-up were included.

\section{Study intervention and control group}

Following delivery of the drug to our ICU, a group of consecutive patients received angiotensin II (Giapreza; La Jolla Pharmaceutical, San Diego, CA, USA) infusion under compassionate use. The drug has been approved by both the EMA and the US FDA. All patients received angiotensin II at ICU admission as vasopressor dose when needing vasopressor therapy or at low dose prophylaxis if vasopressor therapy was not needed. When utilised as a vasopressor, angiotensin II was used in addition to norepinephrine, and when used at low dose, there was the possibility to increase the dose if shock developed. All patients received venous thromboembolic events prophylaxis. Patients in the control group never received angiotensin II and always received venous thromboembolic events prophylaxis.

The control group was made up of consecutive invasively ventilated patients treated before angiotensin II introduction in one of several COVID-19 ICUs and of consecutive invasively ventilated patients admitted to an adjacent COVID-19 ICU where angiotensin II was not made available. In both ICUs, patients were under the care of the same team of doctors and nurses, who rotated across the various
COVID-19 ICUs during the pandemic. The first patient with COVID-19 acute respiratory syndrome was admitted to the ICU on 25 February 2020. During the following weeks, the total number of available ICU beds increased from 28 to 72 (54 of which were dedicated to patients with COVID-19 in seven different ICUs). ${ }^{13}$

\section{Data collection}

Medical records were used for data collection. We obtained data on contact exposure, onset of symptoms and presenting symptoms, medical history and ongoing medications at time of symptoms onset, daily clinical and laboratory data, treatment data, and outcome data. All data were collected by trained investigators independent from the clinical teams. Before analysis, an extensive round of data cleaning was performed by a dedicated data manager, together with clinicians, to check for accuracy.

\section{Outcomes}

All outcomes reported in this study are exploratory in nature and related to the following markers of organ system function:

- mean arterial pressure and norepinephrine dose for the cardiovascular system;

- $\mathrm{PaO}_{2} / \mathrm{FiO}_{2}$ ratio for the respiratory system;

- urinary output, serum creatinine, and use of RRT for the renal system;

- C-reactive protein level for the inflammatory arm of the immune system;

- lactate for the metabolic system;

- elevated liver enzymes for the hepatic system;

- platelets for the coagulation and bone marrow system; and

- clinical thromboembolic complications for the coagulation system.

Additional exploratory analyses included the following clinical outcomes:

- the composite of failure to be discharged alive from the ICU at day 28 or death;

- hospital mortality at day 28;

- duration of mechanical ventilation at day 28; and

- hospital length of stay at day 28.

Safety was evaluated by assessment of development of complications until the completion of follow-up (the complete definitions of the complications are available in the Online Appendix).

\section{Statistical analysis}

A convenience sample was considered for this analysis, with consecutive patients included until the 28 days of follow-up. No missing data for any of the outcomes are present in the 


\section{ORIGINAL ARTICLES}

dataset; therefore, all analyses were complete case analyses without imputation. Continuous variables are presented as median and interquartile range (IQR) and categorical variables as number and percentage. Baseline and clinical characteristics of the patients were compared among the groups using Fisher exact tests and Wilcoxon rank sum tests. The development of complications is presented as unadjusted odds ratios (ORs) from generalised linear models considering binomial distribution. Daily data are compared using mixed effects quantile models accounting for repeated measures, with "day" as continuous variable and "day and group" (and the interaction between them) as fixed effect. Quantile models considered $\tau=0.50$ and an asymmetric Laplace distribution. $P$ values were extracted after 1000 bootstrap samples. Overall $P$ values from this analysis represent the overall difference among groups over time, and $P$ values from interaction represent a statistical assessment of whether the trend over time differed among the groups.

Primary and key secondary outcomes were explored and assessed using a Bayesian perspective. The analysis of key outcomes was done using a Bayesian model considering a Bernoulli distribution or using a Bayesian Cox proportional hazard model, as appropriate. All models were developed using a Markov Chain Monte Carlo simulation with four chains, and considered a burn-in of 1000 iterations, with sampling from a further 10000 iterations for each chain. To monitor convergence, trace plots and the Gelman-Rubin convergence diagnostic (Rhat) were used for all parameters. As is conventional for such analyses, results are presented as hazard ratio (HR) or OR with $95 \%$ credible interval $(\mathrm{Crl})$ and as the probability of minimum clinical benefit. All models were adjusted by key prognostic variables at baseline (age, presence of diabetes, and oxygen saturation measured by pulse oximetry $\left.\left[\mathrm{SpO}_{2}\right]\right)$.

Hospital length of stay and duration of ventilation were assessed under the frequentist approach using subdistribution HR derived from a Fine-Gray competing risk model, with death before the event treated as competing risk and presented in cumulative incidence plots.

It was expected that the effect of angiotensin II would be influenced by the presence of renal dysfunction. Therefore, in the present study, we assessed the different effect of the drug in the subgroup of patients with abnormal serum creatinine at admission (defined as creatinine $>1.10 \mathrm{mg} / \mathrm{dL}$ in females and $>1.20 \mathrm{mg} / \mathrm{dL}$ in males). To explore the potential heterogeneity of treatment effect among these subgroups, a Bayesian binomial model was applied and the posterior distribution was sampled using Markov Chain Monte Carlo simulations. Results are displayed through the probability distribution of HR or OR for the subgroups, and as the probability of a higher benefit in a specific subgroup. Since no previous information about the impact of angiotensin II on COVID-19 is available, all analyses used non-informative flat priors, to have the posteriors completely dominated by the likelihood (reflecting the data).

Due to the nature of the study, and since the sample size was small and the number of events was low, all analyses should be considered exploratory and hypothesisgenerating only. All analyses were conducted in R v.3.6.3 (R Foundation). ${ }^{14}$

\section{Results}

\section{Population}

From 25 February to 7 May 2020, we treated 99 patients with COVID-19 who received mechanical ventilation and completed their follow-up to 28 days. Of these, 46 patients received angiotensin $\|$ and 53 did not (controls). Patient characteristics were balanced at baseline. Most patients were male, the median age was 62 years (IQR, 54-69 years), and $>35 \%$ had obesity (Table 1). The most frequent comorbidities were hypertension and diabetes. The use of ACE inhibitors or angiotensin II receptor blockers was common. Most of the patients treated with angiotensin II received it as rescue vasopressor. Overall, on day one, the median maximum angiotensin II dose used was $5.0 \mathrm{ng} /$ $\mathrm{kg} / \mathrm{min}$ (IQR, 5.0-20.0 $\mathrm{ng} / \mathrm{kg} / \mathrm{min}$ ), but it was higher in patients receiving it as vasopressor (median, $20.0 \mathrm{ng} / \mathrm{kg} /$ $\mathrm{min}$ [IQR, $5.0-20 \mathrm{ng} / \mathrm{kg} / \mathrm{min}]$ v $5.0 \mathrm{ng} / \mathrm{kg} / \mathrm{min}$ [IQR, $5.0-5.0 \mathrm{ng} / \mathrm{kg} / \mathrm{min}]$; median difference, $11.34[95 \% \mathrm{Cl}$, 3.56-19.13]; $P=0.004$ ).

The median time from symptoms to hospital admission and ICU admission was 7.0 (IQR, 4.0-10.0 days) and 10.0 days (IQR, 7.0-14.0 days) respectively (Online Appendix, S1 table). At hospital admission, fever was present in $56.4 \%$ of patients, median $\mathrm{SpO}_{2}$ was $92 \%$ (IQR, 84-96\%), and median respiratory rate was 30 breaths per minute (IQR, 25-36 breaths per minute). All characteristics were similar among the groups (Table 1 and Online Appendix, S1 table).

\section{Exploratory physiological outcomes}

Over the first 3 days, the mean arterial pressure was significantly higher in patients receiving angiotensin II (median difference, 9.05; 95\% Cl, 1.87-16.22; $P=0.013$ for group comparison over time) compared with controls, but the norepinephrine dose was similar (Online Appendix, $\mathrm{S} 1$ figure and $\mathrm{S} 2$ table).

The $\mathrm{PaO}_{2} / \mathrm{FiO}_{2}$ ratio was also significantly higher during the first 7 days in patients receiving angiotensin II (median difference, $23.17 ; 95 \% \mathrm{Cl}, 3.46-42.88 ; P=0.021$ for group 
ORIGINAL ARTICLES

Table 1. Baseline characteristics of the patients according to the use of angiotensin II

\begin{tabular}{|c|c|c|c|}
\hline & Angiotensin II & No angiotensin II & $P$ \\
\hline Total number of patients & 46 & 53 & \\
\hline Median age, years (IQR) & $62(54-69)$ & $62(53-68)$ & 0.653 \\
\hline Sex, male & $41(89.1)$ & $42(79.2)$ & 0.274 \\
\hline Median body mass index, ${ }^{*} \mathrm{~kg} / \mathrm{m}^{2}(\mathrm{IQR})$ & $27.7(25.9-30.5)$ & $27.7(24.8-32.3)$ & 0.910 \\
\hline Healthy weight & $4 / 28(14.3 \%)$ & $7 / 34(20.6 \%)$ & 0.427 \\
\hline Overweight & $16 / 28(57.1 \%)$ & $13 / 34(38.2 \%)$ & \\
\hline Obesity class 1 & $5 / 28(17.9 \%)$ & $9 / 34(26.5 \%)$ & \\
\hline Obesity class 2 & $2 / 28(7.1 \%)$ & $5 / 34(14.7 \%)$ & \\
\hline Obesity class 3 & $1 / 28(3.6 \%)$ & $0 / 34(0.0 \%)$ & \\
\hline \multicolumn{4}{|l|}{ Coexisting disorder } \\
\hline Hypertension & $19 / 41(46.3 \%)$ & $23 / 46(50.0 \%)$ & 0.682 \\
\hline Diabetes & $11 / 41(26.8 \%)$ & $5 / 43(11.6 \%)$ & 0.100 \\
\hline Coronary artery disease & $4 / 41(9.8 \%)$ & $2 / 45(4.4 \%)$ & 0.418 \\
\hline Cardiac arrhythmias & $3 / 41(7.3 \%)$ & $4 / 45(8.9 \%)$ & 0.999 \\
\hline Cerebrovascular disease & $1 / 41(2.4 \%)$ & $1 / 44(2.3 \%)$ & 0.999 \\
\hline Chronic respiratory disease $^{\dagger}$ & $1 / 41(2.4 \%)$ & $0 / 44(0.0 \%)$ & 0.482 \\
\hline Asthma & $1 / 41(2.4 \%)$ & $3 / 44(6.8 \%)$ & 0.617 \\
\hline Chronic obstructive pulmonary disease & $1 / 41(2.4 \%)$ & $1 / 44(2.3 \%)$ & 0.999 \\
\hline Chronic neurological disease ${ }^{\ddagger}$ & $2 / 40(5.0 \%)$ & $0 / 44(0.0 \%)$ & 0.224 \\
\hline Moderate/severe chronic kidney disease ${ }^{\S}$ & $3 / 40(7.5 \%)$ & $3 / 43(7.0 \%)$ & 0.999 \\
\hline Solid tumour & $2 / 39(5.1 \%)$ & $1 / 42(2.4 \%)$ & 0.606 \\
\hline Tobacco smoker & & & 0.999 \\
\hline - Current & $1 / 34(2.9 \%)$ & $1 / 32(3.1 \%)$ & \\
\hline - Former & $2 / 34(5.9 \%)$ & $1 / 32(3.1 \%)$ & \\
\hline \multicolumn{4}{|l|}{ Medications on chronic use } \\
\hline Angiotensin-converting enzyme inhibitors & $6 / 43(14.0 \%)$ & $6 / 42(14.3 \%)$ & 0.999 \\
\hline Angiotensin II receptor blockers & $6 / 43(14.0 \%)$ & $6 / 42(14.3 \%)$ & 0.999 \\
\hline Calcium channel blockers & $6 / 43(14.0 \%)$ & $2 / 42(4.8 \%)$ & 0.265 \\
\hline$\beta$-blockers & $8 / 43(18.6 \%)$ & $6 / 42(14.3 \%)$ & 0.771 \\
\hline Vitamin $\mathrm{K}$ antagonists & $0 / 43(0.0 \%)$ & $1 / 43(2.3 \%)$ & 0.999 \\
\hline Novel oral anticoagulants & $1 / 43(2.3 \%)$ & $0 / 43(0.0 \%)$ & 0.999 \\
\hline Anti-arrhythmic & $2 / 43(4.7 \%)$ & $3 / 43(7.0 \%)$ & 0.999 \\
\hline Aspirin & $10 / 44(22.7 \%)$ & $5 / 44(11.4 \%)$ & 0.256 \\
\hline Other antiplatelets & $2 / 43(4.7 \%)$ & $1 / 43(2.3 \%)$ & 0.999 \\
\hline Statins & $7 / 43(16.3 \%)$ & $3 / 44(6.8 \%)$ & 0.196 \\
\hline Corticosteroids & $0 / 43(0.0 \%)$ & $3 / 44(6.8 \%)$ & 0.241 \\
\hline \multicolumn{4}{|l|}{ Use of angiotensin II } \\
\hline Vasopressor & $26(56.5 \%)$ & na & na \\
\hline Low dose & $20(43.5 \%)$ & na & na \\
\hline
\end{tabular}

$\mathrm{IQR}=$ interquartile range; na = not applicable. Percentages may not total 100 because of rounding. * Body mass index is calculated dividing the kilograms by the square of the height in meters. † Excluding asthma and chronic obstructive pulmonary disease. $\neq$ Excluding dementia and cerebrovascular disease. § Receiving dialysis, after kidney transplant, uraemia or creatinine $>3 \mathrm{mg} / \mathrm{dL}$

comparison over time) (Figure 1 and Online Appendix, S2 table). After adjustment for potential confounding effect of different levels of positive end-expiratory pressure and use of prone positioning among the groups, the use of angiotensin II was still associated with a higher $\mathrm{PaO}_{2} / \mathrm{FiO}_{2}$ ratio (median difference, $19.76 ; 95 \% \mathrm{Cl},-0.13$ to 39.65 ; $P=0.051$ for group comparison over time).
Daily laboratory data are shown in the Online Appendix, S3 table. There were no differences between angiotensin II patients and controls for lactate, urinary output, serum creatinine, and C-reactive protein levels (Figure 2). However, there was a decrease in markers of liver dysfunction in patients treated with angiotensin II (Online Appendix, S4 table). 


\section{ORIGINAL ARTICLES}

Figure 1. Daily ventilation variables according to use of angiotensin II
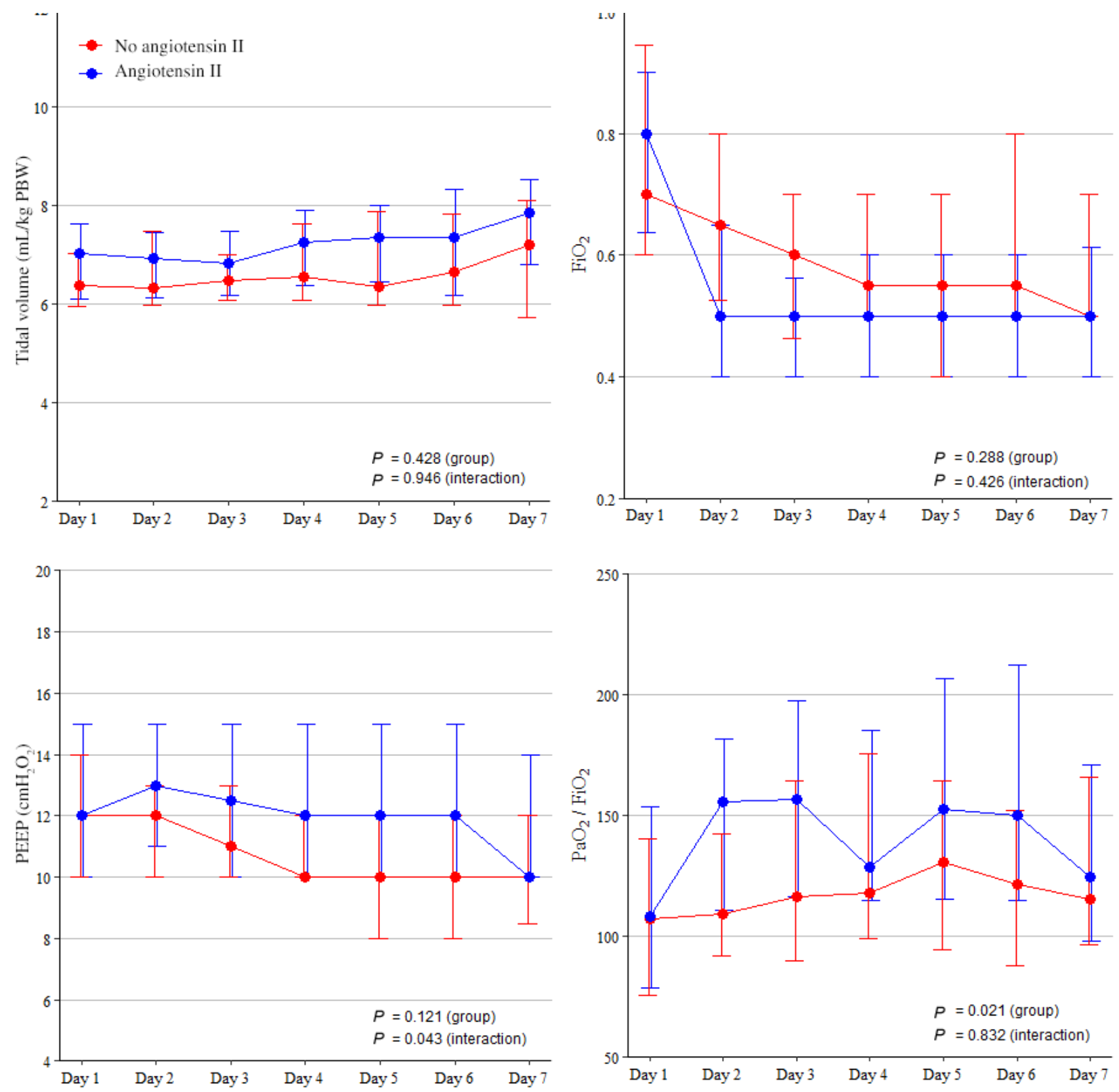

$\mathrm{FiO}_{2}=$ fraction of inspired oxygen; $\mathrm{PaO}_{2}=$ arterial partial pressure of oxygen; $\mathrm{PBW}=$ predicted body weight; $\mathrm{PEEP}=$ positive end-expiratory pressure. Data are median and interquartile range. $P$ values are calculated from a mixed-effect quantile model considering $\alpha=0.50$; an asymmetric Laplace distribution and $P$ values were extracted after 1000 bootstrap samples. $P$ values from this analysis represent the overall difference among groups over time, and $P$ values from interaction represent if the trend differs among the groups over time.

Moreover, in the angiotensin II therapy group, there was no difference in platelet count (Online Appendix, S3 table) and there were two episodes $(4.3 \%)$ of clinically relevant pulmonary embolism compared with four $(7.5 \%)$ in the control group. There was one episode of clinically relevant limb ischaemia in the angiotensin II group versus three such episodes in the control group.
Overall, seven angiotensin II patients and ten nonangiotensin II patients received RRT with an adjusted OR of $0.87(95 \% \mathrm{Crl}, 0.25-2.97)$ and a $58.9 \%$ probability of benefit in terms of avoiding RRT with angiotensin II. However, such probability was higher $(95.7 \%)$ in patients with abnormal renal function at baseline with an adjusted OR of 0.23 (95\% Crl, 0.04-1.23) (Figure 3). 


\section{ORIGINAL ARTICLES}

Figure 2. Daily clinical and laboratory variables according to use of angiotensin II
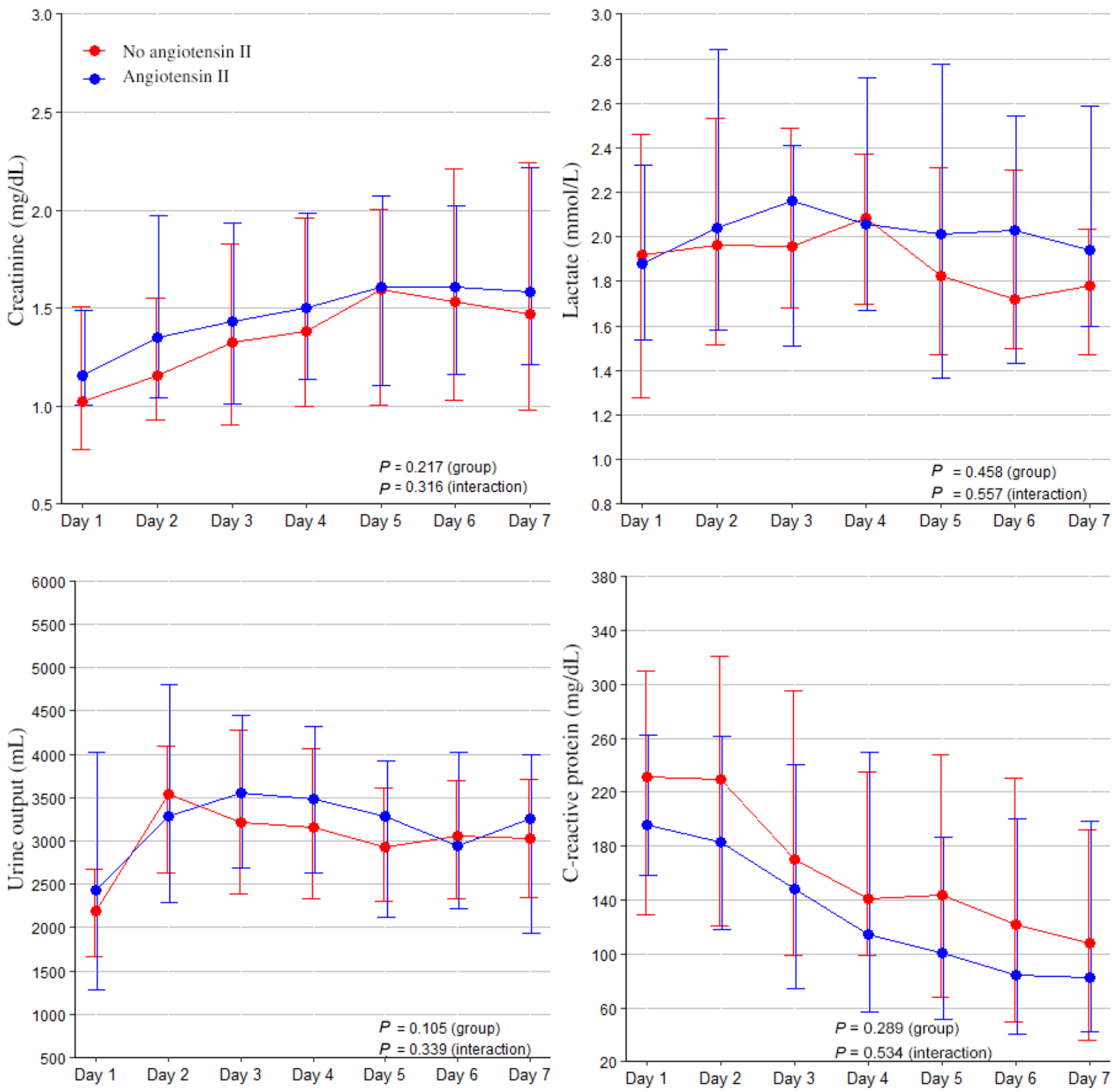

Data are median and interquartile range. $P$ values calculated from a mixed-effect quantile model considering $\alpha=0.50$; an asymmetric Laplace distribution and $P$ values were extracted after 1000 bootstrap samples. $P$ values from this analysis represent the overall difference among groups over time, and $P$ values from interaction represent if the trend differs among the groups over time.

\section{Exploratory clinical outcomes}

Younger age and higher baseline $\mathrm{SpO}_{2}$ values were present in patients who were discharged alive at day 28, and diabetes was twice as common among patients who were not discharged alive from the ICU (Online Appendix, S5 table).
Results for exploratory clinical outcomes are presented in Table 2 and in the Online Appendix, S2 figure. After accounting for confounders and considering all patients together, compared with patients not receiving angiotensin II, the probability of angiotensin II patients showing a reduced risk of developing the composite primary outcome of failure to be discharged or death at day 28 was $58.1 \%$, 


\section{ORIGINAL ARTICLES}

Figure 3. Heterogeneity of treatment effect according to presence of abnormal renal function at admission and the use of renal replacement therapy
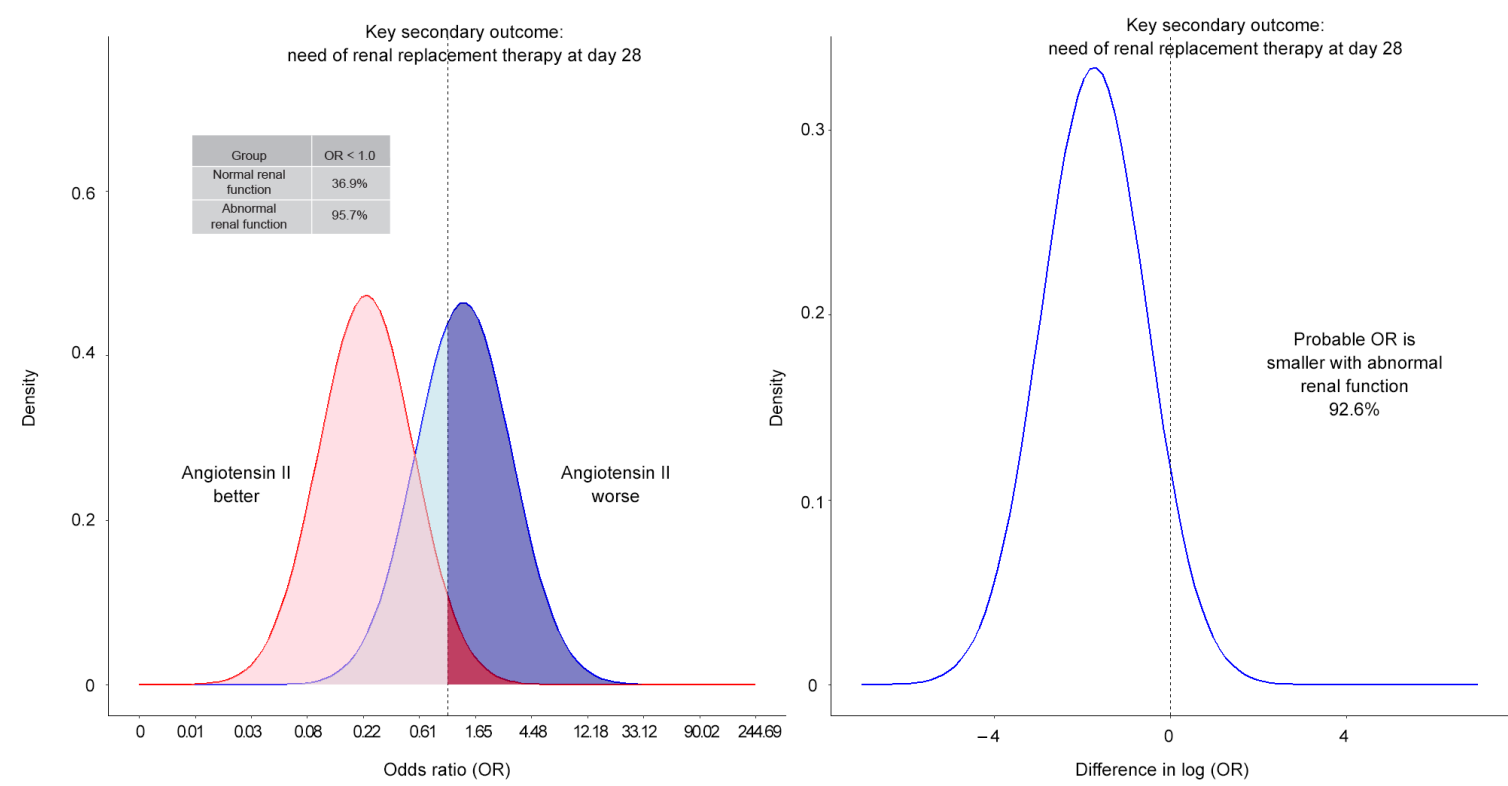

The light red area represents where angiotensin II is beneficial and the dark red area represents where angiotensin II is harmful. Abnormal renal function is defined as creatinine $>1.10 \mathrm{mg} / \mathrm{dL}$ in females and $>1.20 \mathrm{mg} / \mathrm{dL}$ in males. The blue line represents patients with normal renal function and the red line represents patients with abnormal renal function.

with a Bayesian adjusted effect estimate OR of $0.91(95 \%$ $\mathrm{Crl}, 0.38-2.18)$. For all patients, similar overall results were seen for hospital mortality, need for RRT, duration of mechanical ventilation or hospital length stay (Table 2).

\section{Discussion}

\section{Key findings}

In a cohort of almost 100 consecutive invasively ventilated patients with COVID-19, we conducted an exploratory study of the effects of introducing angiotensin II therapy on markers of organ function. We found that angiotensin II therapy was associated with increased mean arterial pressure levels and $\mathrm{PaO}_{2} / \mathrm{FiO}_{2}$ ratios; decreased risk of liver dysfunction; no effect on urinary output, creatinine, lactate, platelet count, thromboembolic complications, and C-reactive protein levels; and limited overall effects on RRT use. However, angiotensin II therapy was associated with a high probability of decreased RRT use among patients with elevated serum creatinine at admission. Finally, angiotensin II was not associated with harm in relation to mortality, length of invasive mechanical ventilation, thromboembolic events, and length of stay in hospital.

\section{Relationship with previous studies}

This is the first controlled evaluation of angiotensin II as adjunctive treatment in ventilated patients with COVID-19 pneumonia. However, a recent case series showed evidence of increased $\mathrm{PaO}_{2} / \mathrm{FiO}_{2}$ ratios over time ${ }^{9}$ and our study confirms this effect on oxygenation. Angiotensin II has been previously found to increase blood pressure in catecholamine refractory vasodilatory shock in a randomised placebocontrolled trial, 6,7 which was also shown in our study.

The use of angiotensin II has been previously reported as beneficial in patients with markedly abnormal renal function at randomisation. ${ }^{9}$ Our findings of a potential decrease in RRT use in COVID-19 patients with abnormal renal function at admission support such previous reports.

Moreover, our study results indicate a possible protective liver function effect, which is novel and requires confirmation in future studies. Our findings of no effect on platelet count or thromboembolic complications are reassuring given previous theoretical concerns. ${ }^{15,16}$ The lack of adverse effects on lactate and the C-reactive protein levels are novel and also reassuring.

In a 2020 research letter, ${ }^{12}$ concern was raised that angiotensin II may contribute to mortality. However, this report only involved six patients and did not have any controls. Our findings, which did not show an adverse effect on mortality, time on mechanical ventilation or duration of hospital stay compared with controls, provide a degree of reassurance. 
Table 2. Primary, key secondary outcomes and secondary outcomes according to the use of angiotensin II

\begin{tabular}{|c|c|c|c|c|c|}
\hline Clinical outcomes & Angiotensin II & No angiotensin II & $\begin{array}{l}\text { Effect estimate } \\
(95 \% \mathrm{Crl} \text { or } \mathrm{Cl})^{*}\end{array}$ & $\begin{array}{l}\text { Probability of } \\
\text { benefit }^{\dagger}\end{array}$ & $\begin{array}{l}\text { Absolute } \\
\text { difference } \\
(95 \% \mathrm{Crl})^{\ddagger}\end{array}$ \\
\hline Total number of patients & 46 & 53 & & & \\
\hline $\begin{array}{l}\text { Failure to be discharged or } \\
\text { death at day } 28\end{array}$ & $28(60.9 \%)$ & $31(58.5 \%)$ & $0.91(0.38-2.18)^{\S}$ & $58.1 \%$ & $\begin{array}{c}2.35 \% \\
(-16.69 \% \text { to } \\
21.08 \%)\end{array}$ \\
\hline $\begin{array}{l}\text { Hospital mortality at day } \\
28\end{array}$ & $15(32.6 \%)$ & $14(26.4 \%)$ & $0.99(0.61-1.60)^{n}$ & $51.4 \%$ & $\begin{array}{c}5.98 \% \\
(-11.57 \% \text { to } \\
23.54 \%)\end{array}$ \\
\hline $\begin{array}{l}\text { Need for renal } \\
\text { replacement therapy at } \\
\text { day } 28\end{array}$ & $7(15.2 \%)$ & $10(18.9 \%)$ & $0.87(0.25-2.97)^{\S}$ & $58.9 \%$ & $\begin{array}{c}-3.55 \% \\
(-17.71 \% \text { to } \\
11.05 \%)\end{array}$ \\
\hline $\begin{array}{l}\text { Median duration of } \\
\text { mechanical ventilation at } \\
\text { day } 28 \text {, days (IQR) } \\
\text { In survivors }\end{array}$ & $16.0(10.6-28.0)$ & $18.6(9.9-28.0)$ & $1.07(0.56-2.07)^{* *}$ & $0.830^{t+}$ & $\begin{array}{c}-2.76(-8.89 \\
\text { to } 3.35)\end{array}$ \\
\hline $\begin{array}{l}\text { Median hospital length of } \\
\text { stay at day 28, days (IQR) } \\
\text { In survivors }\end{array}$ & $28.0(16.2-28.0)$ & $28.0(20.0-28.0)$ & $0.82(0.23-2.93)^{* *}$ & $0.760^{+\dagger}$ & $\begin{array}{c}0.92(-2.13 \text { to } \\
3.97)\end{array}$ \\
\hline
\end{tabular}

$\mathrm{Crl}=$ credible interval; ICU = intensive care unit; IQR = interquartile range. Percentages may not total 100 because of rounding. * For the median duration of mechanical ventilation at day 28 and the median hospital length of stay at day 28 , the estimate is reported with the $95 \%$ Cl; for all others, it is reported with $95 \% \mathrm{Crl}$. + Defined as hazard ratio (HR) or odds ratio (OR) $<1.00$. ₹ Unadjusted. § Effect estimate is OR (95\% Crl) from a Bayesian model with a Bernoulli distribution adjusted by age, diabetes status, and oxygen saturation measured by pulse oximetry $\left(\mathrm{Spo}_{2}\right)$ at admission. ๆ Effect estimate is $\mathrm{HR}(95 \%$ Crl) from a Bayesian Cox proportional hazard model adjusted by age, diabetes status, and $\mathrm{SpO}_{2}$ at admission. ** Effect estimate is the subdistribution $\mathrm{HR}$ $\left(95 \% \mathrm{Crl}\right.$ ) from an unadjusted Fine-Gray competing risk model, with death before the event as competing risk adjusted by age, diabetes status, and $\mathrm{SpO}_{2}$ at admission. †† $P$ value.

\section{Implications of study findings}

Our study results imply that angiotensin II is an effective vasopressor agent in patients with COVID-19 pneumonia. They also imply that it is associated with greater improvements in the $\mathrm{PaO}_{2} / \mathrm{FiO}_{2}$ ratio in such patients and with decreased liver enzyme release. Furthermore, they imply no adverse effects on lactate, platelets, or thromboembolic complications, mortality, or duration of mechanical ventilation and hospital stay. Finally, our findings imply that angiotensin II may decrease the risk of RRT in COVID-19 patients with abnormal kidney function at ICU admission.

\section{Strengths and limitations}

The strengths of our study relate to the presence of a control population, and the availability of a detailed dataset of physiological and clinical observations. In addition, this is the first controlled study of any vasopressor in patients with COVID-19. The comparative safety and efficacy of other vasopressors (norepinephrine, epinephrine or vasopressin) remain untested. Moreover, our findings have implications in relation to the use of angiotensin $\|$ in patients with abnormal creatinine at baseline.

We acknowledge several limitations to our study: it is single-centre, limited in size, not randomised, and openlabel in design. Such characteristics may raise concerns about external validity, limited statistical power, and potential performance and selection bias. The lack of differences in urinary output and serum creatinine may appear contradictory given a possible effect on RRT use. However, RRT was started early and would have markedly affected serum creatinine measurements, thus making their interpretation problematic. In addition, urinary output was easily modified by the use of diuretics, which have been used widely in patients with COVID-19 as a lung protective strategy and for which we do not have data. A randomised controlled trial would have been desirable. However, in the setting of the COVID-19 outbreak in Italy, the conduct of a double-blind randomised controlled trial 


\section{ORIGINAL ARTICLES}

was logistically impossible. Moreover, we had detailed information on both patients and controls and similarities were strong and baseline imbalances negligible. In addition, we applied appropriate statistical analyses and took major confounding factors and baseline imbalances into account in the adjusted analyses. Finally, we did not demonstrate a survival advantage. However, this study was markedly underpowered to detect such an effect.

\section{Conclusion}

In a controlled exploratory assessment on markers of organ function during angiotensin II therapy in invasively ventilated patients with COVID-19 pneumonia, we found that angiotensin II increased blood pressure and $\mathrm{PaO}_{2} / \mathrm{FiO}_{2}$ ratio and carried an adjusted $>95 \%$ estimated probability of decreasing RRT use in patients with abnormal baseline serum creatinine at admission. Moreover, it carried no signal of other organ function or clinical harm. These findings imply the need to continue investigations of this agent in a larger population and a multicentre setting.

\section{Competing interests}

No relevant disclosures.

\section{Author details}

Alberto Zangrillo ${ }^{1,2}$

Sergio Colombo'

Anna Mara Scandroglio ${ }^{1}$

Evgeny Fominskiy'

Marina Pieri ${ }^{1}$

Maria Grazia Calabrò ${ }^{1}$

Paolo Federico Beccaria ${ }^{1}$

Nicola Pasculli ${ }^{1}$

Francesca Guzzo'

Maria Rosa Calvi ${ }^{1}$

Antonella Cipriani ${ }^{1}$

Chiara Sartini ${ }^{1}$

Pasquale Nardelli ${ }^{1}$

Alessandro Ortalda ${ }^{1}$

Gaetano Lombardi ${ }^{1}$

Marianna Sartorelli ${ }^{1}$

Giacomo Monti ${ }^{1}$

Andrea Assanelli ${ }^{3}$

Moreno Tresoldi ${ }^{4}$

Lorenzo Dagna2,5

Stefano Franchini ${ }^{6}$

Ary Serpa Neto ${ }^{7,8}$

Rinaldo Bellomo 7,9,10

Giovanni Landoni ${ }^{1,2}$
1 Department of Anesthesia and Intensive Care, IRCCS San Raffaele Scientific Institute, Milan, Italy.

2 Vita-Salute San Raffaele University, Milan, Italy.

3 Unit of Hematology and Bone Marrow Transplantation, IRCCS San Raffaele Scientific Institute, Milano, Italy.

4 Unit of General Medicine and Advanced Care, IRCCS San Raffaele Hospital, Milan, Italy.

5 Unit of Immunology, Rheumatology, Allergy and Rare Diseases (UnIRAR), IRCCS San Raffaele Hospital, Vita-Salute San Raffaele University, Milano, Lombardia, Italy.

6 Emergency Department, IRCCS San Raffaele Scientific Institute, Milan, Italy.

7 Australian and New Zealand Intensive Care Research Centre (ANZIC-RC), School of Public Health and Preventive Medicine, Monash University, Melbourne, VIC, Australia.

8 Department of Critical Care Medicine, Hospital Israelita Albert Einstein, São Paulo, Brazil.

9 Faculty of Medicine, University of Melbourne, Melbourne, VIC, Australia.

10 Department of Intensive Care, Austin Hospital, Melbourne, VIB, Australia.

Correspondence: landoni.giovanni@hsr.it

\section{References}

$1 \mathrm{Wu} \mathrm{Z}$, McGoogan JM. Characteristics of and important lessons from the coronavirus disease 2019 (COVID-19) outbreak in China: summary of a report of 72314 cases from the Chinese Center for Disease Control and Prevention. JAMA 2020; 323: 1239-42.

2 Zangrillo A, Beretta L, Scandroglio AM, et al. Characteristics, treatment, outcomes and cause of death of invasively ventilated patients with COVID-19 ARDS in Milan, Italy. Crit Care Resusc 2020; 22: 200-11.

3 Guan WJ, Ni ZY, Hu Y, et al. Clinical characteristics of coronavirus disease 2019 in China. N Engl J Med 2020; 382: 1708-20.

4 Hoffmann M, Kleine-Weber $H$, Schroeder $S$, et al. SARSCOV-2 Cell Entry Depends on ACE2 and TMPRSS2 and Is Blocked by a Clinically Proven Protease Inhibitor. Cell 2020; 181: 271-80.

5 Wrapp D, Wang N, Corbett KS, et al. Cryo-EM structure of the 2019-nCoV spike in the perfusion conformation. Science 2020; 367: 1260-3

6 Busse LW, Chow JH, McCurdy MT, Khanna AK. COVID-19 and the RAAS - a potential role for angiotensin II? Crit Care 2020; 24: 136-9.

7 Khanna A, English SW, Wang XS, et al. Angiotensin II for the treatment of vasodilatory shock. N Engl J Med 2017; 377: 419-30.

8 Bellomo R, Wunderink RG, Szerlip H, et al. Angiotensin I and angiotensin II concentrations and their ratio in catecholamineresistant vasodilatory shock. Crit Care 2020; 24: 43. 


\section{ORIGINAL ARTICLES}

9 Bellomo R, Forni LG, Busse LW, et al. Renin and survival in patients given angiotensin II for catecholamine-resistant vasodilatory shock. A clinical trial. Am J Respir Crit Care Med 2020; 202: 1253-61.

10 Tumlin JA, Murugan R, Deane AM, et al. Outcomes in patients with vasodilatory shock and renal replacement therapy treated with intravenous angiotensin II. Crit Care Med 2018; 46: 949-57.

11 Zangrillo A, Landoni G, Beretta L, et al. Angiotensin II infusion in COVID19-associated vasodilatory shock: a case series. Crit Care 2020; 24: 227

12 Heinicke U, Adam E, Sonntagbauer M, et al. Angiotensin II treatment in COVID-19 patients: more risk than benefit? A single-center experience. Crit Care 2020; 24: 409.
13 Zangrillo A, Beretta L, Silvani $P$, et al. Fast reshaping of intensive care unit facilities in a large metropolitan hospital in Milan, Italy: facing the COVID-19 pandemic emergency. Crit Care Resusc 2020; 22: 91-4.

14 R Core Team. R: a language and environment for statistical computing. Vienna, Austria: R Foundation for Statistical Computing, 2019. https://www.R-project.org/ (viewed Mar 2021).

15 Drugs.com. Giapreza. https://www.drugs.com/pro/giapreza. html (viewed Mar 2021).

16 Ciceri F, Beretta L, Scandroglio AM, et al. Microvascular COVID-19 lung vessels obstructive thromboinflammatory syndrome (MicroCLOTS): an atypical acute respiratory distress syndrome working hypothesis. Crit Care Resusc 2020; 22: 95-7. 